\title{
Role of urinalysis in patients with lichen planus
}

Simona Roxana Georgescu ${ }^{1}$, Corina-Daniela $\mathrm{Ene}^{2}$, Mircea Tampa ${ }^{1}$, Cristina lulia Mitran ${ }^{3}$, Madalina Irina Mitran ${ }^{3}$, llinca Nicolae ${ }^{3}$

1. "Carol Davila" University of Medicine and Pharmacy, Bucharest, Romania

2. "Carol Davila" Clinical Hospital of Nephrology

3. "Victor Babes"Hospital of Infectious and Tropical Diseases, Bucharest, Romania

Lichen planus (LP) is a complex immunologically mediated disease, which affects the skin and mucous membranes. In medical literature there are few reports on the connection of LP with renal disorders. In this paper we present some findings on the association between LP and renal disorders.

We conducted an observational study on a sample of 77 patients with LP (49 cases of cutaneous LP, 28 cases of oral LP) and 50 healthy controls. The evaluation of study participants included clinical examination and paraclinical investigations.

Compared to controls, in the patients with LP the following quantitative changes were found: a low value of estimated glomerular filtration rate (eGFR) (18.18\%), an elevated albumin/creatinine ratio hyperuricosuria (16.88\%), hypocitraturia (14.28\%), hyperoxaluria (11.68\%), hypercalciuria (10.39\%), hypomagnesiuria (15.58\%), hypernatriuria (19.48\%), leukocyturia (18.18\%), erythrocyturia (14.28\%), bacterial/fungal infections (24.67\%) (Figure 1).

Based on anamnesis, imaging and laboratory tests the following kidney and urinary tract disorders were diagnosed in the patients with LP: diabetic nephropathy (3 cases), nephritis (1 case), membranous glomerulonephritis related to hepatitis $B$ virus (1 case), urolithiasis (8 cases), urethritis (4 cases), pyelonephritis (7 cases), cystitis (5 cases) (Figure 2 ).

No differences were observed between patients with oral LP and cutaneous LP regarding the presence of renal impairment $(p=0.264)$.

\section{References}

1. .Krupaa RJ, Sankari SL, Masthan KMK, Rajesh E. Oral lichen planus: An overview. J. Pharm. Bioallied. Sci. 2015; 7(1):158-161.

2. Are patients with lichen planus really prone to urolithiasis? Lichen planus and urolithiasis. Int Braz J Urol. 2016; 42(3): 571-7.

3. Nogueira PA, Carneiro S, Ramos-e-Silva M. Oral lichen planus: an update on its pathogenesis. International journal of dermatology. 2015;54(9):1005-10.
These findings prove that LP is a condition frequently associated with renal manifestations (a decline of kidney function was identified in 1 of 5 patients with LP). The use of a full panel of investigations in patients with LP may help the dermatologist elaborate a plan of close observation in order to prevent the development of complications in these patients.

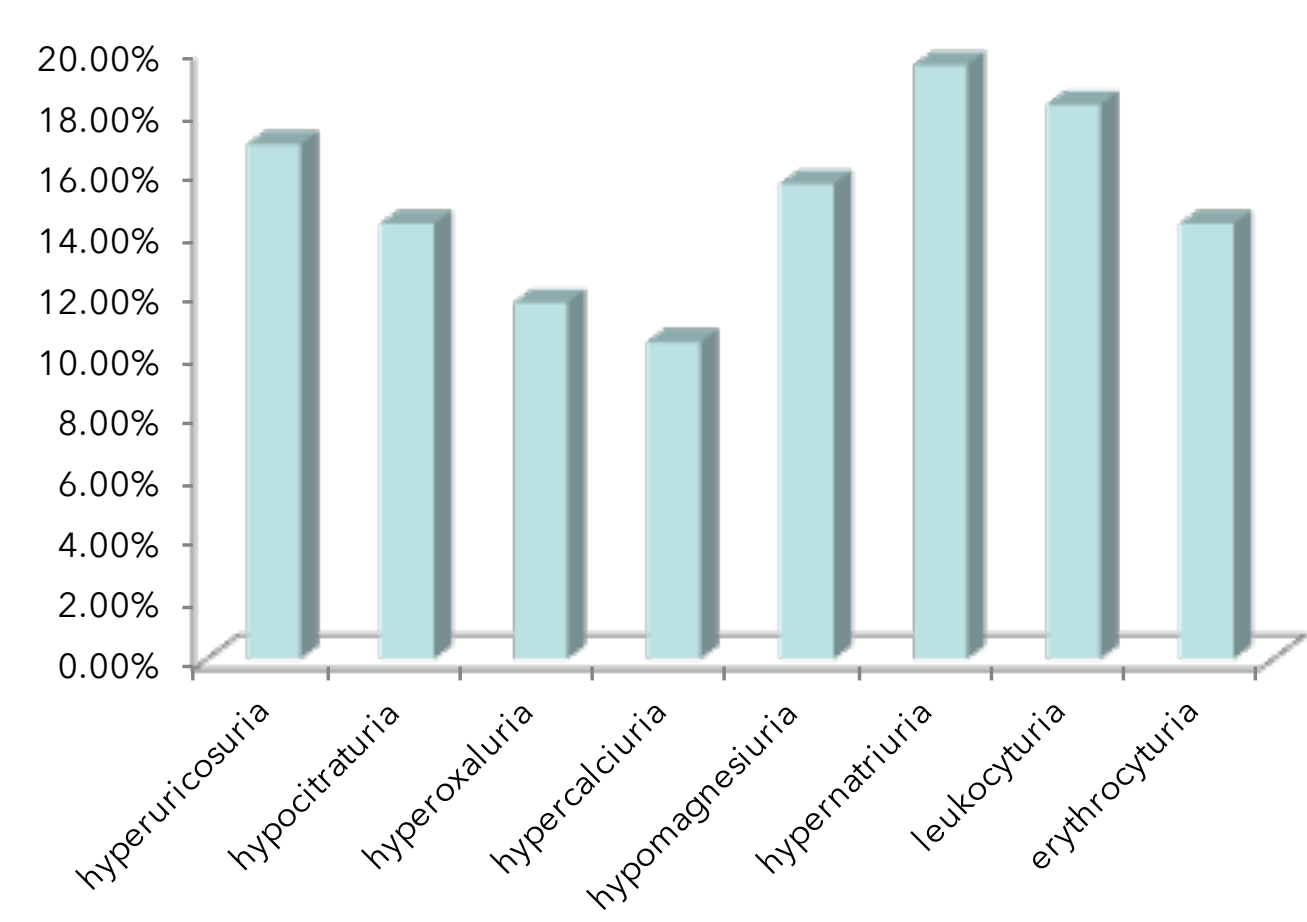

Urinalysis in LP patients

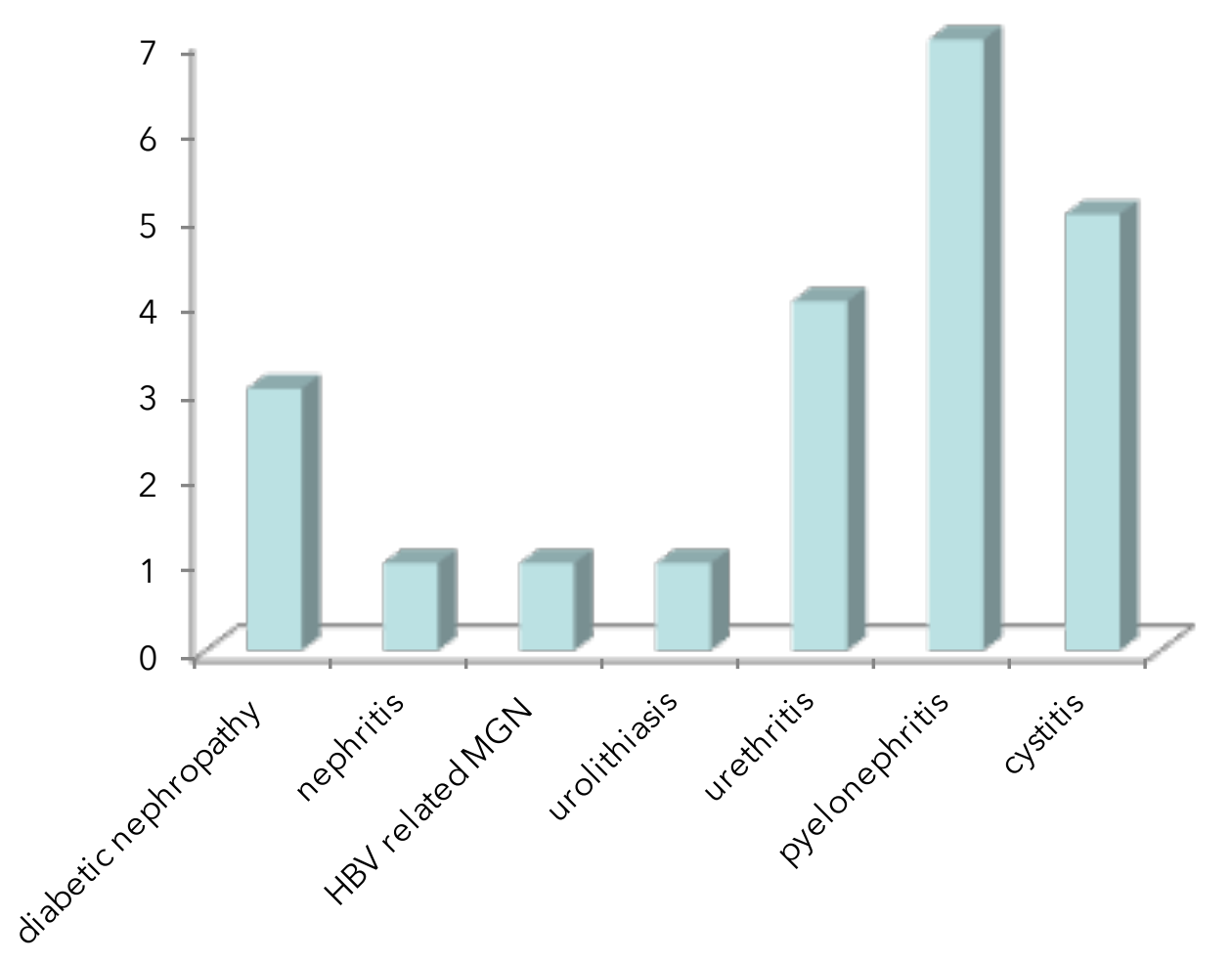

KIdney and urinary tract disorders in LP patients

This work was possible with the financial support of Young Researchers Grant from the Carol Davila University of Medicine and Pharmacy, no. 33884/11.11.2014 